The proposed Charter will have a profound influence on the psychiatric service. On the positive side, it may lead to an improved out-ofhours service. However, by making unrealistic promises, this Charter is setting up the service for fallure. Our most serious concern is that by reinforcing the bias of service provision to those who 'shout loudest' the Charter will further marginalise the seriously mentally ill: would a reclusive psychotic ask for an appointment on a specific day, giving 48 hours notice?

The draft edition of the Charter invited comments before 26 April 1996. We have written to Mr Tony Day of the NHS Executive requesting that the publication of the Charter is delayed until there has been consultation with a wide group of mental health care professionals.

\section{R. DENNY}

Fromeside Clinic, Stapleton, Bristol BS16 1ED

S. FORSHALL

Robert Smith Unit, 12 Mortimer Road, Bristol

BS8 4EX

H. REES

Knowle Clinic, Knowle, Bristol BS4 2UH

\section{Propofol and ECT}

Propofol was introduced as an induction agent for ECT in our hospital last year but, after four months of its use, it was discontinued as seizures were often described by the medical officer giving the ECT as brief or inadequate. Also, the ECT machine had to be set at a higher than average setting using a higher dose of electricity. In the Royal College of Psychiatrists' guidelines (1995) propofol is specifically not recommended for ECT.

It was decided, as an audit topic, to look retrospectively at the last 53 patients who had ECT under either propofol or methohexitone. Clinical outcome after the course of ECT was obtained from case notes. Recorded clinical improvement was rated as marked, moderate or none, based on what was stated in the case notes after the last administered ECT application. The sample included 31 patients who received ECT under methohexitone and 22 patients under propofol.

Duration of seizures was significantly longer with a mean of 25 seconds with methohexitone compared with 18 seconds with propofol $(P<0.01)$. The mean setting of the ECT machine was $226 \mathrm{mQ}$ for ECT given with methohexitone compared with $269 \mathrm{mQ}$ with propofol $(P<0.01)$. There was no evidence to suggest that patients who received ECT under propofol, and despite the significantly shorter seizure duration compared with methohexitone, required additional ECT applications. The mean number of ECTs were
5.1 and 4.8 for methohexitone and propofol induced ECT respectively $(P=6)$. The recorded clinical outcome following the ECT course given under either agent was not significantly different $(P>0.05)$

This may imply that ECT under either anaesthetic was equally effective. Also the similar clinical outcome after ECT given under either agent may suggest that the reduced, visible, seizure duration may be misleading and should not be taken to indicate poor therapeutic effect of ECT. However the retrospective nature of data collection, with non-randomisation, also the possibility that in some cases, the number of ECTs may have been determined in advance by some consultants are flaws of this review which may limit any conclusion that can be made.

Royal Counge of Psychiatrists (1995) The ECT Handbook. London: RCPsych.

E. SALIB, C. BARLOW and J. HARRISON Winwick Hospital, Warrington

\section{Information of interest}

The medical director and representatives of the Association of British Pharmaceutical Industries (ABPI) and representatives of the RCPsych held their second 9-monthly meeting on 29 September 1995. We felt it would be worthwhile to inform members of various points which arose during the meeting concerning patient prescribing and clinical trials.

The pharmaceutical industry will be introducing a procedure to put a leaflet with prescriptions which inform patients about the drugs that are being dispensed under prescription, including their actions and side-effects. The industry will also continue its practice of supporting Continuing Professional Development, medical education, postgraduate meetings and scientific meetings of interest to the profession.

The ABPI has produced a draft contract for pharmaceutical companies to indemnify Trusts and patients who participate in clinical trials. A survey carried out over the past 5 years with the intention of recording the requirement for indemnity payments found that there were only 20 cases out of 415000 patients who participated in clinical trials during the survey period, an incidence of $0.005 \%$. Three-quarters of these came from one clinical trial and there were no court proceedings.

The ABPI can provide any one who is interested with a list of standards they have established for training for both industry funded and nonindustry funded research projects involving the treatment of patients. The public should be 
reassured that routine checks are now carried out in all industry sponsored clinical trials in order to detect made up or fraudulent data.

It is expected that in the not too distant future large multicentre trials will be scrutinised by a lead ethical committee in several centres throughout the UK before being referred to local ethical committees who will have the power only to accept or reject the trial, but not to modify the protocol. The time and cost to develop new drugs has hitherto been delayed by the need to have multiple modifications of protocol procedures because of the large number of committees which individually approved such studies.

It was agreed that as a routine doctors involved in clinical trials should be advised to ensure that they have proper cover from their medical insurance society because the indemnity provided by Trusts may not be sufficient. It might be expected that most pharmaceutical companies would be prepared to pay any increase in insurance which was required for doctors participating in such clinical trials. Pharmaceutical companies cannot be expected to indemnify doctors against their own negligence. For clinical practice this is currently covered by the obligation of the Trust but this may not be so in the case of clinical trials.

\section{S. R. HIRSCH}

Chairman, Psychopharmacology Sub-Committee, RCPsych

\section{Alternatives to district general hospitals?}

Sir: The Department of Health (1996) claims that "Acute treatment does not have to be located on District General Hospital sites ....". Alternatives to district general hospitals are cited and include developments in Stoke-on-Trent. We have consultant responsibility for these innovative beds in Stoke-on-Trent and would view them as complementing district general beds not replacing them.

We each have eight PIR beds in a purpose built bungalow attached to a community mental health resource centre and over a population of 80000 . Conceptually the main use of these beds is the prevention of further deterioration in mental health, short admissions aimed at intervention and respite care. It was also hoped that being local, accessible and not hospital based they would be more user friendly than traditional beds. While the PIR beds meet some of these objectives they cannot fulfil all the roles of traditional district general beds. Some of the problems encountered have been logistical and include out of hours medical cover, provision of pharmacy services, security and nursing staff levels. More importantly, at least $10 \%$ of patients are returned urgently to the district general unit, usually due to deterioration in their mental state or physical condition. Patients are not admitted directly to the PIR beds unless they are known to the service and present no apparent risk of suicide or violence.

At present we are investigating the clinical and cost effectiveness of these beds in comparison with district general beds and until our results are available we would advise caution in the belief that other forms of psychiatric bed provision can replace the district general.

DEPARTMENT OF HEALTH (1996) The spectrum of care. Local services for people with mental health problems. Wetherby: DOH.

RICHARD HODGSON and JED BOARDMAN

The Sutherland Centre, Belgrave Road, Stoke-on-Trent ST3 4PN

\section{Use of Section 17 trial leave}

Sir: I disagree with James et al (Psychiatric Bulletin. 20, 201-204) that it is proper for Section 17 leave to be used in the manner described in their paper. The main advantage that they give for use of Section 17 as opposed to Section 19 is that it ensures the continuing involvement of the local hospital and ensures that patients are transferred back to their hospital at the earliest opportunity. The suggestion, therefore, is that consultant colleagues will not act in the best interest of patients unless there is some external legal requirement for them so to do.

It seems strange that at a time when a patient is at their most distressed and disturbed they are sent to a hospital where they will be under the care of a consultant who is not their Responsible Medical Officer, i.e., a doctor who is not authorised to assess a patient's ability to consent to medical treatment, to alter their medical treatment, to authorise trial leave and so on.

The very word 'grant' implies that the leave is with the approval of, if not at the request of, the patient. I am unsure if this would always apply when a patient is transferred from an ordinary hospital to an Regional Secure Unit.

Finally, it is usually considered appropriate to recall a patient from leave when it is necessary in the interest of the patient's health or safety or for the protection of others because the patient has failed in some way while on leave. The notion of recalling a patient from leave on the basis that the leave has been successful is somewhat unusual.

\section{A. S. ZIGMOND}

Leeds Community \& Mental Health Services, Inkley LS29 5AQ 